\title{
Modelagem da Propagação de Ondas Sísmicas em Meios Viscoelásticos descritos pelo Modelo de Burger
}

\author{
Luiz Fernando da Silva ${ }^{1}$, Marcos Antonio Barsottelli Botelho ${ }^{2}$, Marco Antonio Cetale Santos ${ }^{3}$, Djalma Manoel Soares Filho ${ }^{4}$ \\ ${ }^{1}$ UNESA e Departamento e Geologia e Geofísica/Universidade Federal Fluminense \\ ${ }^{2}$ CPGG/Universidade Federal da Bahia \\ ${ }^{3}$ Departamento de Geologia e Geofísica/Universidade Federal Fluminense \\ ${ }^{4}$ CENPES/PETROBRAS
}

\section{Copyright 2014, SBGf - Sociedade Brasileira de Geofísica}

Este texto foi preparado para a apresentação no VI Simpósio Brasileiro de Geofísica, Porto Alegre, 14 a 16 de outubro de 2014. Seu conteúdo foi revisado pelo Comitê Técnico do VI SimBGf, mas não necessariamente representa a opinião da SBGf ou de seus associados. É proibida a reprodução total ou parcial deste material para propósitos comerciais sem previa autorização da SBGt.

\section{Resumo}

Os estudos de modelagem são de grande relevância na investigação e prospecção de petróleo. Um dos processos de investigação do meio geológico é o da utilização de um modelo viscoelástico (Modelo de Maxwell, Kelvin-Voigt, Solido Padrão, Burger etc) na implementação numérica da equação da onda e assim obter informações de subsuperfície (snapshots, sismogramas). Uma das vantagens de usar esses modelos é que eles incorporam a atenuação do meio neste processo de modelagem. Nosso trabalho consiste em utilizar um modelo viscoelástico mais realístico (Modelo de Burger) e dessa forma recuperar informações do meio 0 mais fiel possível. Inicialmente foi implementado o Modelo de Maxwell para estudos e testes de algoritmo. Posteriormente foi validada uma nova metodologia de implementação de modelos viscoelásticos na modelagem sísmica. Aplicou-se essa nova metodologia utilizando o Modelo Viscoelástico de Burger e os resultados estão de acordo com a teoria prevista.

\section{Introdução}

A investigação de um meio sobre o qual se quer obter informação é realizada por meio de uma perturbação (uma onda gerada artificialmente) e então verificamos como o meio reagiu a essa perturbação. Essa análise do comportamento do meio pode ser por intermédio do campo de velocidades, do tensor das tensões e das deformações, do traço sísmico etc. Na modelagem via processamento de sinal, o traço sísmico apresenta uma grande relevância para geração e análise dos dados. No processo de modelagem através de modelos elásticos e viscoelásticos, as equações constitutivas e dinâmicas do meio é que exercem uma grande importância. $\mathrm{O}$ procedimento adotado para modelar é o de acoplamento das: equações constitutivas do meio (Lei de Hooke); variáveis de memória (equação constitutiva hereditária, memória evanescente, tensão e deformação em função do tempo, função Fluência e função Relaxação);

Equação dinâmica do meio (equação de Euler, equação da onda), equação de Christofell etc. O objetivo principal do acoplamento dessas equações é descrever de maneira exata o comportamento do meio devido a uma perturbação. Os trabalhos publicados pelos pesquisadores dessa área ao longo dos anos e nos dias atuais, basicamente buscam melhorar esse acoplamento entre as equações para obter resultados mais precisos, Clay \& McNeil, 1955, Angona, 1960, Altermam \& Karal, 1968, Alford et al., 1974, Day \& Minster, 1984, Dablain, 1986, Emmerich \& Korn, 1987; Carcione et al., 1988a; Carcione et al., 1988b; Carcione et al., 1988c, Casula \& Carcione, 1992. Fatores tais como: dispersão e atenuação, são relevantes na pesquisa de modelagem e por isso têm-se muito interesse na investigação desses fenômenos. Os mecanismos de atenuação e dispersão do meio são simulados através da parametrização do fator da qualidade $Q$ modelado pelo, (no caso viscoelástico), modelo viscoelástico utilizado (por exemplo: Modelo de Maxwell( uma mola e um amortecedor em série) ou Modelo de Kelvin-Voigt (uma mola e um amortecedor em paralelo, etc), das equações constitutivas (Lei de Hooke: que descreve as tensões e deformações sofridas pelo meio devido a essa perturbação), dos mecanismos de memória evanescente:que são descritos através da Equação Constitutiva Hereditária, onde as tensões são relacionadas no tempo com as deformações e as deformações são relacionadas no tempo com as tensões. Esse processo de convolução gera, respectivamente as funções relaxação e fluência. Ou seja, o processo segue alguns passos que são direcionados conforme o modelo elástico ou viscoelástico adotado; então prossegue-se com os cálculos para encontrarmos as equações que regem o meio através do modelo adotado e depois segue com uma implementação por um método numérico.

Utilizou-se o modelo de 04 parâmetros ( Modelo de Burger) no processo de modelagem. O modelo escolhido apresenta aspectos dos modelos viscoelásticos (Maxwell, Kelvin-Voigt e Sólido Linear Padrão) mais utilizados, e cada um deles é descrito pelo Modelo de Burger sob certas condições do meio (valores de densidades e viscosidades). Por apresentar essas características, o modelo proposto deve 
recuperar informações melhores do imageamento de subsuperfície.

\section{Metodologia/ Problema Investigado}

A anelasticidade depende, geralmente, de um grande número de mecanismos físicos, os quais podem ser modelados por diferentes teorias micro estruturais. As propriedades físicas do meio real podem ser representadas por modelos mecânicos. Tais modelos mecânicos são representados por molas (desprezando-se o peso nenhum efeito inercial estará presente, representam o sólido elástico) e amortecedores (Pistão em um cilindro preenchido com um fluido viscoso, representando a parte viscoelástica do sistema e relacionada como um dos mecanismos de perda). Esses modelos são utilizados para modelar as propriedades físicas do meio real e conforme o modelo adotado, todo um processo de modelagem do meio é desenvolvido. A Figura 1 descreve alguns desses modelos.

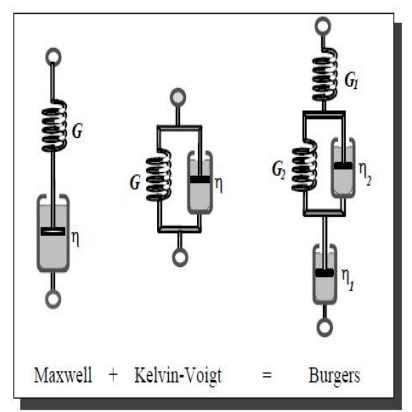

Figura 1 - Modelo de Maxwell, Kelvin-Voigt e o Modelo

Proposto.

Cada modelo tem seu fator de qualidade e o mesmo é implementado no processo de modelagem.

A teoria da mecânica do contínuo, a simulação numérica e a propagação de ondas são o eixo principal do desenvolvimento do projeto . O método de diferenças finitas é utilizado na implementação dos algoritmos de representação do modelo, Levander, 1988, Courant et al., 1928, Carcione, 1990, Carcione et al., 2004. O modelo inicial para teste foi 0 de Maxwell (onda $P$ ) e foi implementado como se segue:

- Modelo Viscoelástico de Maxwell adotado para a construção do processo de modelagem do meio.

- a implementação do algoritmo de diferença finita de marcha no tempo ( equação do movimento em 2D);

- A validação do Método de Inserção Direta - MID (nova metodologia proposta), que insere a solução da equação diferencial do Modelo de Maxwell (a função relaxação $\sigma(\mathrm{t})$ ) no passo de tempo de execução do algoritmo.

- $\quad$ A utilização do MID na implementação do Modelo de Burger na modelagem viscoelástica.
- a implementação numérica utilizada é a de diferença finitas com peso ( no espaço com aproximação de quarta ordem e segunda ordem no tempo).

\section{Resultados}

Os testes de implementação de modelagem numérica e algoritmo mostraram-se satisfatórios e apresenta-se alguns resultados obtidos com o algoritmo implementado. Como resultados preliminares tem-se algumas figuras geradas pelo algoritmo de teste que utilizou o Modelo de Maxwell e teoria de variáveis de memória (Figuras 2 e 3). Apresentam-se alguns snapshots e sismogramas em tempo. O modelo geológico usado foi o mais simples possível (modelo de duas camadas separadas por interface horizontal, $X=500 \mathrm{~m}$ e $Z=500 \mathrm{~m}$ ).

$V p_{1}=3000 \mathrm{~m} / \mathrm{s} ; \rho_{1}=2400 \mathrm{~kg} / \mathrm{m}^{3} ; E_{1}=\rho_{1}\left(\mathrm{Vp}_{1}\right)^{2}=21,6$ GPa na camada 1 e $V p_{2}=2300 \mathrm{~m} / \mathrm{s} ; \rho_{2}=2000 \mathrm{~kg} / \mathrm{m}^{3} ; E_{2}$ $=\rho_{2}\left(\mathrm{Vp}_{2}\right)^{2}=10,58 \mathrm{GPa}$ na camada 2 .

O modelo foi discretizado usando uma malha quadrada em $d x=5 \quad m \quad e \quad d z=5 \quad m$. Apresentam-se alguns sismogramas que levam em consideração a variação de parâmetros tais como: viscosidade e seus respectivos $Q$ do fator da qualidade e seus efeitos de atenuação.

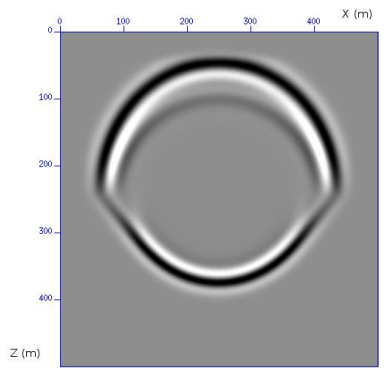

Figura 2 - Instantâneo do campo de onda $P$ no instante $\mathrm{t}=0,4 \mathrm{~s}$. Ondas: direta, refletida e transmitida. Tiro na posição $(250 \mathrm{~m}, 230 \mathrm{~m})$. Metodologia de variáveis de memória.

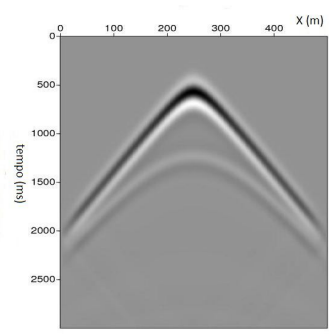

Figura 3 - Sismograma com tempo total de amostragem $\mathrm{t}=3,0$ s. Ondas: direta e refletida. Metodologia de variáveis de memória.

As Figuras 4, 5 e 6 são relativas a validação da metodologia MID. Isto é: comparativas entre a teoria de variáveis de memória e a metodologia proposta (MID). 
Utilizou-se o modelo geológico de duas camadas citado anteriormente.
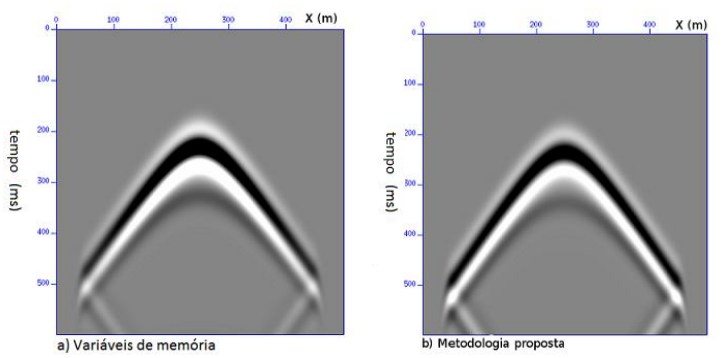

Figura 4 - Sismogramas a e b para comparação de metodologias. Frequência de pico de $10 \mathrm{~Hz}$ e Fator da Qualidade $\mathrm{Q}=10$.
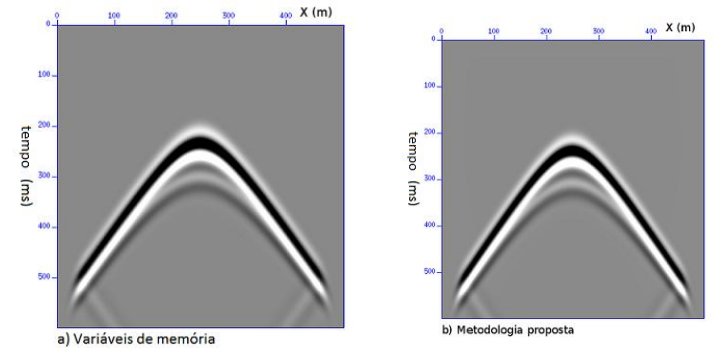

Figura 5 - Sismogramas a e b para comparação de metodologias. Frequência de pico de $15 \mathrm{~Hz}$ e Fator da Qualidade $\mathrm{Q}=100$.

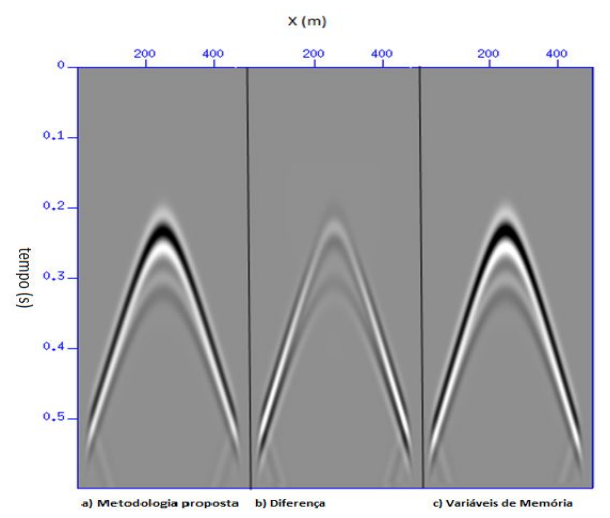

Figura 6 - Comparação entre as metodologias (sismogramas a e c) e a diferença entre elas é representada pelo sismograma $b$.

Como exemplo numérico de comparação entre as duas metodologias, têm-se os dados de valores máximos e mínimos de amplitude dos sismogramas apresentados na Figura 6. Variáveis de Memória - VM: Valor máximo $\left(8,512667 \times 10^{4} \mathrm{~Pa}\right)$, Valor mínimo (-)6,013875 x $\left.10^{4} \mathrm{~Pa}\right)$. MID: Valor máximo $\left(9,614372 \times 10^{4} \mathrm{~Pa}\right)$, Valor mínimo ($6,782262 \times 10^{4} \mathrm{~Pa}$ ). Erro percentual para os máximos de amplitude: VM (12,9 \%), MID (11,5\%). Erro percentual para os mínimos de amplitude: VM (0,13\%), MID $(0,11$ $\%$ ). A diferença entre os erros percentuais (VM e MID) para os máximos de amplitude é de $1,4 \%$ _ e para os mínimos de amplitude é de 0,02\%.
Na aplicação do MID utilizou-se o Modelo de Burger e o modelo geológico com armadilha anticlinal (Figura 7) e seus parâmetros são conforme a tabela 1.

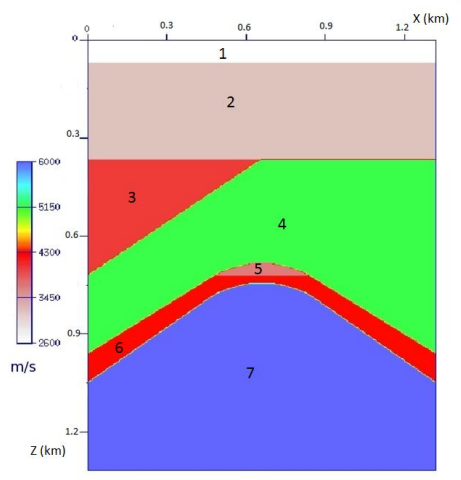

Figura 7 - Modelo geológico com armadilha anticlinal.

\begin{tabular}{|c|c|c|c|c|}
\hline Canada (i) & Densitade $\rho_{1}\left(\frac{k_{m}}{m}\right)$ & Velocitade Compresikinal $\mathrm{V}_{\mathrm{Pl}}(\mathrm{m})$ & Mádula Yaung $\mathrm{E}_{\mathrm{i}}(G \mathrm{pa})$ & Viscositade $m_{n}\left(10^{10} p a . s\right)$ \\
\hline 1 & 2400 & 2600 & 16,22 & 16,22 \\
\hline 2 & 2530 & 3200 & 25,90 & 25,90 \\
\hline 3 & 2670 & 4000 & 42,72 & 42,72 \\
\hline 4 & 2850 & 5200 & 77,06 & 77,06 \\
\hline 5 & 2610 & 3650 & 34,77 & 34,77 \\
\hline 6 & 2720 & 4300 & 50,29 & 50,29 \\
\hline 7 & 2960 & 6000 & 106,56 & 106,65 \\
\hline
\end{tabular}

Tabela 1 - Parâmetros do modelo geológico com armadilha anticlinal mostrado na Figura 7.

Na modelagem utilizando o Modelo de Burger, apresentase resultados com espaçamento do grid de $D X=D Z=4 \mathrm{~m}$, frequência da fonte de $30 \mathrm{~Hz}$ e um modelo geológico com armadilha anticlinal (conforme Figura 7) (Carcione, 1993). Os sismogramas foram gerados através da metodologia proposta, que é a de inserir a informação de atenuação do meio no passo de tempo do programa. São apresentados sismogramas para os meios elástico e viscoelástico, respectivamente, e Uz é o deslocamento na direção z. Os dados do modelo geológico, com armadilha anticlinal utilizado no Modelo de Burger, foram obtidos conforme a seguir: o Modelo de Burguer é a soma dos Modelos de Maxwell e Voigt, considera-se as camadas do modelo geológico adotado, obedecendo ao Modelo de Maxwell e, na parte relacionada ao Modelo de Voigt, é arbitrado um valor para o módulo de Young e calculada a viscosidade. $O$ cálculo de valor da viscosidade para a parte relacionada ao Modelo de Maxwell é dado pela Eq. $\eta_{i}=(Q / w) E_{i}$. Nesta modelagem, os dados estão relacionados a $Q=300$ e $w=30 \mathrm{~Hz}$, conforme Figuras 8, 9 e 10. Na parte do Modelo de Burger relacionada ao Modelo de Voigt tem-se: escolhese um valor para o módulo de Young e calcula-se a viscosidade obedecendo ao modelo considerado. Dessa forma, obtém-se: $\mathrm{E}_{8}=7,4 \mathrm{GPa}$ e $\eta_{8}=\mathrm{E}_{8}(\mathrm{Q} / \mathrm{w})=0,822$ $\times 10^{5}$ Pa.s. Essa parte do modelo é aplicada a todas as camadas do modelo geológico adotado. 


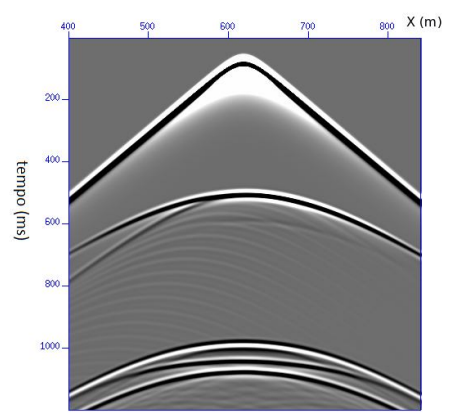

Figura 8 - Sismogramas com tempo total de amostragem de $1,2 \mathrm{~s}$. Frequência de pico de $30 \mathrm{~Hz}$. Tiro na posição $(620 \mathrm{~m}, 23 \mathrm{~m})$. Geofones distribuídos ao longo da linha (X $\mathrm{m}, 12 \mathrm{~m}$ ). Sismograma Uz, caso elástico.

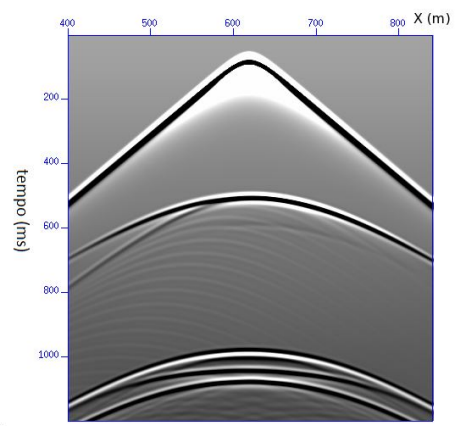

Figura 9 -.Sismogramas com tempo total de amostragem de $1,2 \mathrm{~s}$. Frequência de pico de $30 \mathrm{~Hz}$. Tiro na posição (620 m, $23 \mathrm{~m})$. Geofones distribuídos ao longo da linha (X m, $12 \mathrm{~m}$ ). Sismograma Uz, caso viscoelástico.

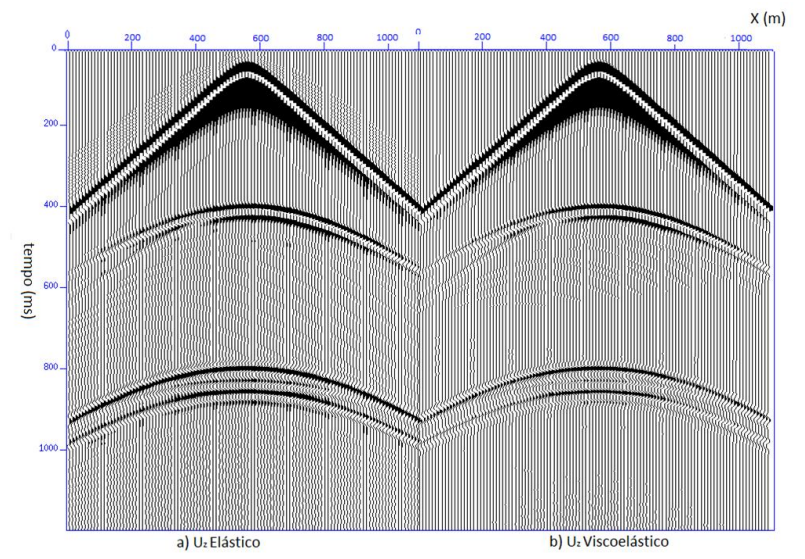

Figura 10 - Sismogramas com tempo total de amostragem de 1,2 s. Frequência de pico de $30 \mathrm{~Hz}$. Tiro na posição $(570 \mathrm{~m}, 23 \mathrm{~m})$. Geofones distribuídos ao longo da linha

(X m, 22 m). Sismograma: (a) Uz elástico e (b) Uz viscoelástico.

\section{Discussão e Conclusões}

A modelagem sísmica implementada através de modelos viscoelásticos incorpora a atenuação do meio, sendo portanto muito importante na geração de sismogramas sintéticos mais próximos da realidade. Considerar o meio como viscoelástico no processo de modelagem é necessário para inserção de um dos mecanismos de atenuação do meio geológico considerado. O método numérico utilizado para discretizar e implementar o modelo de propagação de ondas sísmicas foi o de diferenças finitas e a literatura já mostrou que ele é eficiente neste tipo de modelagem. Dessa forma, o método de diferenças finitas foi utilizado, neste trabalho, na elaboração dos algoritmos implementados para descrever a propagação de ondas sísmicas em meios viscoelásticos. Os algoritmos elaborados, com a metodologia de variáveis de memória e com a metodologia proposta, foram testados e posteriormente confrontam-se os seus resultados. Comparam-se sismogramas em tempo, do modelo geológico de duas camadas (conforme seção de validação da metodologia proposta) e os resultados mostraram-se compatíveis com a teoria existente (variáveis de memória). O método de inserção direta $(M I D)$ incorpora o $\sigma_{\mathrm{kl}}(\mathrm{t})$, no passo de tempo do algoritmo, como uma informação de estado de tensão do meio geológico no tempo. $O$ algoritmo da metodologia MID levou três segundos a menos para gerar os mesmos sismogramas (Laptop Neo PC A2250, 4 GB Memória RAM, Processador Pentium T4200). Fez-se uma diferença entre os sismogramas gerados pela teoria de variáveis de memória e pela metodologia MID: os resultados estão apresentados nas Figuras 4,5 e 6 . Posteriormente, implementou-se o Modelo de Burger com a metodologia proposta, pois ela implementa mais facilmente modelos viscoelásticos no processo de modelagem sísmicas e aplicou-se num modelo geológico complexo: trapa anticlinal típica de acúmulo de hidrocarbonetos. Os resultados mostraram-se satisfatórios e verificou-se que o efeito da atenuação apresenta-se mais forte, principalmente nos refletores mais profundos.

A metodologia proposta apresenta-se como uma contribuição no processo de modelagem sísmica que utiliza modelos viscoelásticos (devido à facilidade de implementação) e dessa forma, pode ser usada na implementação de outros modelos que ainda não foram testados. O Modelo de Burger utilizado nesta pesquisa é mais apropriado para o processo de investigação e inédito neste tipo de descrição de propagação de ondas sísmicas em meios viscoelásticos. Este trabalho de tese contribui para geração de resultados mais próximos da realidade e também como mais um método a ser usado na modelagem sísmica.

\section{Agradecimentos}

O primeiro autor gostaria de agradecer a Capes pela bolsa de estudos de doutorado e a Petrobras/ANP e UFF pela bolsa de pós-doutorado e ao CPGG pelo apoio. Os autores gostariam de agradecer a PETROBRAS ao suporte dado ao Laboratório de Imageamento Sísmico e Inversão Sísmica (ISIS) do Departamento de Geologia e Geofísica da Universidade Federal Fluminense e ao CPGG da Universidade Federal da Bahia. 


\section{Referências}

Alford, R.M., Kelly, K.R \& Boore, D.M. (1974) Accuracy of finite-diference modeling of acoustic wave propagation, Geophysics, 39:834-842.

Alterman, Z. \& Karal, F.C. Jr. (1968) Propagation of elastic waves in layered media by finite diferences methods. Bulletin of Seismological Society of America, 58:367-398.

Angona, F.A. (1960) Two dimensional modeling and its application to seismic problems, Geophysics, 25:480-482.

Carcione, J.M. (1990) Wave propagation in anisotropic linear viscoelastic media: theory and simulated wave fields, Geophys. Journal International, 101:739-750.

Carcione, J.M., Poletto, F. \& Gei, D. (2004) 3-D wave simulation in anelastic media using the Kelvin-Voigt constitutive equation. Computational Physics Journal, 196:282-297.

Carcione, J.M., Koslof, D. \& Koslof, R. (1988a) Wave propagation simulation in a linear viscoacoustic medium. Geophysical Journal, 93:393-407.

Carcione, J.M., Koslof, D. \& Koslof, R. (1988b) Wave propagation simulation in a linear viscoelastic medium. Geophysical Journal, 95:597-611.

Carcione, J.M., Koslof, D. \& Koslof, R. (1988c) Viscoacoustic wave propagation simulation in the earth. Geophysics, 53:769-777.

Carcione, J.M., (1993) Seismic modeling in viscoelastic media. Geophysics, 58:110-120.

Casula, G. \& Carcione, J.M. (1992) Generalized Mechanical Model analogies of linear viscoelastic behaviour. Bollettino Di Geofsica Teorica, Ed. Applicata. Cerjan, C.; Koslof, D.; Koslof, R. \& Reshef, M. (1985) A nonrefecting boundary condition for discrete acoustic and elastic wave equations. Geophysics, 50:705-708.

Clay, C.S. \& McNeil, H. (1955) An amplitude study on a seismic model. Geophysics, 20:700-733.

Courant, R., Friedrichs, K. \& Lewy, H. (1928) On The Partial Diference Equations of Mathematical Physics. IBM Journal - March 1967, 215-234.

Dablain, M. A. (1986) The application of high-order diferencing to the escalar wave equation. Geophysics, 51:54-66.

Day, S. M. \& Minster, J. B. (1984) Numerical simulation of attenuaded wavefelds using a Paddé approximant method. Geophys. J. R. Astr. Soc., 78:105-118.
Emmerich, H. \& Korn, M. (1987) Incorporation of attenuation into time-domain computations of seismic wave felds. Geophysics, 52:1252-1264.

Levander, A.R. (1988) Four-order finite diference P-SV seismograms. Geophysics, 53:1425-1436. 